Editorial

\title{
Cancer stem cell research: the future direction
}

\section{Editorial}

Scientists have tried for many years to understand cancer development in the context of therapeutic strategies. The recognition that cancers may rely on "cancer stem cells" that share the self-renewal feature of normal stem cells has changed the perspective with regard to new approaches for treating the disease. In recent years, biomedical research has revealed extraordinary diversity in the molecular basis of cancers, confusing attempts to find common underlying bases for therapeutic purposes. However, all clinically significant cancers share at least one common characteristic: excessive proliferation of affected cells, resulting in two main therapeutic approaches for combating cancerous cells. Differentiation therapy attempts to induce differentiation in cancer cells, as highly differentiated cells rarely divide. Alternatively, destruction therapy attempts to thwart malignant proliferation, since rapidly proliferating cells have poorly differentiated phenotypes. Both approaches have resulted in limited successes for certain cases, but neither approach completely cures cancer, targeting downstream cancerous cells, rather than the source.

Stem cells divide to produce two daughter cells: one exact copy, for continuous self-renewal and a second 'progenitor' cell, which differentiates into mature cells that give rise to all the cell lineages in corresponding tissues. Stem cells have greater opportunity to accumulate genetic mutations, due to the combined ability for continuous self-renewal and the longer life span as compared with their cellular progeny. Any of the numerous signaling pathways that stem cells use to control normal self- renewal can be deregulated to produce cancer stem cells. The stem cell model of cancer suggests that accumulation of mutations in stem cells - or their early progenitor cells can result in the deregulation of self-renewal, leading to extensive proliferation abilities of the "cancer stem cells" and subsequent tumorigenesis.

The cancer stem cell model was first demonstrated in 1994 in John Dick's lab, where scientists effectively induced tumorigenesis in severe combined immunodeficient (SCID) mice via the use of rare, leukemia-initiating stem cells purified from patients suffering from acute myelogenous leukemia (AML). Almost a decade later, Michael Clarke's lab demonstrated the presence of breast cancer stem cells while Peter Dirk's lab demonstrated the equivalent in brain cancer.

\author{
Volume I Issue I - 2014
}

\author{
Mohannad AL-Saghir,' Burouj Ajlouni² \\ 'Department of Biological Sciences, Ohio University Zanesville, \\ USA \\ ${ }^{2}$ Fisher College of Business, The Ohio State University, USA
}

Correspondence: Mohannad AL-Saghir, Department of Biological Sciences, Ohio University Zanesville, USA, Tel 54084I5108,Email al-saghi@ohio.edu

Received: April 15, 2014 | Published: April 23, 2014

These cancer stem cells are rare (about 1 in 250,000 tumor cells), but are the only cells with the capability to produce a new tumor. The remaining majorities of cancer cells within established tumor are unable to maintain the tumor and are not capable of tumorigenesis. This scarcity of tumorigenic cancer stem cells with metastatic ability has important implications for cancer therapeutics, as these cells are insensitive to traditional therapeutics and are able to regrow tumors, even after the bulk of non-tumorigenic cancerous cells are removed. This provides an explanation for regrowth of tumor mass after removal of the bulk of the tumor and the lack of cure for certain types of cancers.

The cancer stem cell model has thus changed the perspective of cancer research and allows novel therapeutic strategies to advance. Clearly, the solution to effective therapies is to target the rare cells from whence the cancer originates, rather than all the cells of the tumor, in an effort to eliminate the disease itself and prevent these cancer stem cells from repopulating the tumor once the bulk is killed off. As cancer research advances, identifying and eliminating these cancer stem cells will become the basis for effective therapy, targeting the underlying basis of the disease and resulting in a possible cure for cancer.

\section{Acknowledgements}

None.

\section{Conflict of interest}

The author declares no conflict of interest. 